\title{
Fully Metal-Coated Scanning Near-Field Optical Microscopy Probes with Spiral Corrugations for Superfocusing under Arbitrarily Oriented Linearly Polarised Excitation
}

\author{
Valeria Lotito • Urs Sennhauser • Christian Hafner • \\ Gian-Luca Bona
}

Received: 23 October 2010 / Accepted: 1 February 2011 /Published online: 17 February 2011

(C) The Author(s) 2011. This article is published with open access at Springerlink.com

\begin{abstract}
We study the effect of a spiral corrugation on the outer surface of a fully metal-coated scanning near-field optical microscopy (SNOM) probe using the finite element method. The introduction of a novel form of asymmetry, devoid of any preferential spatial direction and covering the whole angular range of the originally axisymmetric tip, allows attaining strong field localization for a linearly polarised mode with arbitrary orientation. Compared to previously proposed asymmetric structures which require linearly polarised excitation properly oriented with respect to the asymmetry, such a configuration enables significant simplification in mode injection. In fact, not only is the need for the delicate procedure to generate radially polarised beams overcome, but also the relative alignment between the linearly polarised beam and the tip modification is no longer critical.
\end{abstract}

Keywords Scanning near-field optical microscopy. Surface plasmons $\cdot$ Apertureless probes $\cdot$ Finite element method $\cdot$ Superfocusing

V. Lotito $(\bowtie) \cdot$ C. Hafner $\cdot$ G.-L. Bona

Department of Information Technology and Electrical Engineering, ETH Zurich,

Gloriastrasse 35,

8092 Zurich, Switzerland

e-mail: Valeria.Lotito@empa.ch

V. Lotito $\cdot$ U. Sennhauser $\cdot$ G.-L. Bona

Electronics/Metrology Laboratory, EMPA,

Swiss Federal Laboratories for Materials Science and Technology,

Ueberlandstrasse 129 ,

CH-8600 Dübendorf, Switzerland

\section{Introduction}

Scanning near-field optical microscopy (SNOM) has represented a major step in nano-optics as an instrument to study nanostructure properties thanks to its ability to shed light on topographical and optical features with subwavelength resolution, by using the near-field interactions between the tip and the sample. A crucial role in such a mechanism is of course played by the probe itself, independently of whether it is used as a local illuminator to excite the sample, whose response is then collected in the far field (illumination mode), as a local collector of the near-field response of the sample, broadly irradiated with a far field excitation (collection mode), or both (illumination/ collection mode) [1].

The importance of the probe justifies the intensive search for optimised structures [2-4], whose analysis has also been favoured by the flourishing of a vast range of numerical methods giving a glimpse into nanoscale mechanisms [5, 6]. The so-called aperture probes have been at the heart of SNOM since its very beginning [7, 8]. Typically excited with $\mathrm{HE}_{11}$ linearly polarised modes, they exhibit low resolution and poor throughput, together with asymmetric near-field distribution [9]. An interesting alternative is given by apertureless fully metal-coated probes. If externally illuminated by a focusing lens or using a prism-based total internal reflection configuration, they allow the achievement of high resolution, albeit limited by a strong background. Fortunately, such a shortcoming can be avoided by resorting to internal back excitation [9-12].

The behaviour of fully metal-coated tips depends on the input polarisation. The three fundamental modes supported by the input aperture are a pair of orthogonal linearly polarised eigenmodes, followed by a radially polarised one. The probe behaviour under diversely polarised excitation 
has been carefully investigated in both numerical and experimental studies [9-11, 13-17]. Strong field localization with peak amplitude much higher than the one achievable with linearly polarised modes has been observed in case of radially polarised excitation. Such a nanofocusing effect, of utmost importance for SNOM applications, has been explained as the result of the energy transfer from a waveguide mode (WGM) to the surface plasmon polaritons (SPPs) propagating towards the apex, occurring when their respective wave vectors are matched [14]. More detailed analyses have later emphasised the role played in such intercoupling mechanisms by both inner and outer SPP modes sustained at the metal/fibre and air/metal interfaces, respectively [10]. If linearly polarised modes are injected at the probe input aperture, the excited surface plasmons will cancel out at the tip apex because of the opposite polarities on the opposite sides of the tip. On the other hand, under radially polarised injection, the excited surface plasmons will interfere constructively at the very end of the tip due to the rotational symmetry of the input polarisation and the probe $[13,15,18]$. These mechanisms are responsible for the high field confinement in case of radially polarised excitation compared to the linearly polarised case. The eventual outcome of this process is the creation of an ultrasmall hot spot in the region close to the tip apex in the former situation, as opposed to broader and weaker twolobed electric field intensity distributions for the latter one.

In spite of its potential attractiveness for SNOM set-ups, radially polarised excitation requires an awkward procedure extremely sensitive to misalignments, which could impair all the benefits inherent in its use [19]. However, field confinement under a more straightforward linearly polarised excitation can be achieved by introducing an asymmetry in the originally axisymmetric structure. This route has been followed in several studies, assessing the effect of both unintentional asymmetries, in form of defects in the metal coating, and intentional modifications, like slits, an oblique cut (stripping off both the metal coating and the core of the original tip) and asymmetric corrugations on the metal surface [20-23]. All these modifications introduce an asymmetry along one specific direction. Hence, field localization occurs only for the linearly polarised mode that is oriented in the direction of the asymmetry. In other words, given $z$ the direction of the probe axis, if the geometric modification determines an asymmetry along, say, the $x$ direction, then the $x$ linearly polarised mode undergoes significant shrinkage with respect to the axisymmetric structure and also peak value enhancement for a proper choice of the characteristics of the modification; vice versa, the $y$ linearly polarised mode will not be dramatically affected by the modification, as no asymmetry is present along the $y$ direction, and will maintain a weaker, broader and substantially two-lobed distribution. The field localiza- tion stems from an interplay of different coupling mechanisms between the WGM and SPPs, the inner and outer SPPs, the linearly polarised mode and the radial one. Similar considerations hold for asymmetries that do not involve the geometry, rather the illumination, as is the case for the offset apertured-metal-coated dielectric apertureless structure, where a localised hot spot is obtained by using incident light polarised linearly in the direction perpendicular to the interface between the base of the tip and the offset aperture adjacent to the tip base [24]. All these forms of asymmetries exhibit their own axis of symmetry and represent "directional" asymmetries that, of course, cannot guarantee field localization for arbitrarily oriented linearly polarised modes. Even if such modifications entail a considerable simplification in the injection procedures, they still require alignment between the linearly polarised mode and the asymmetry itself. Therefore, it would be desirable to attain superfocusing effects for arbitrarily oriented linearly polarised excitation, by using an "adirectional" asymmetry.

In this paper, we analyse the effects of a spiral corrugation on the outer metal surface of a fully metalcoated probe. The spiral intrinsically fits the specification of lack of directionality and offers an interesting case study to scrutinise the feasibility of the concept of orientationinsensitive field localization. Although such a structure looks challenging for fabrication, it could represent an interesting starting point for more practical structures. In the first section, we will show the possibility to get field localization for two orthogonal linearly polarised modes. Then, we will vary the mutual orientation of the linearly polarised modes with respect to the spiral and compare the results with those achievable with directional asymmetries. We will show that, while the directional asymmetries give rise to orientation-dependent behaviour, the adirectional asymmetry brings about performance significantly less sensitive to variations in the mutual orientation, which makes the tip even more robust against misalignments.

\section{Probe with Spiral Winding}

The structure we have examined is based on a spiral metal corrugation on the outer metal coating of the originally axisymmetric structure, in a form likely to cover the entire angular range and to create a fully asymmetric structure along any spatial direction. We used a finite element based software, Comsol Multiphysics, to carry out our threedimensional (3D) analysis. The sketch of the 3D structure and of its projection on the $x y$ plane is reported in Fig. 1.

The initial probe is made up of a silica core $(n=1.5)$ surrounded by an aluminium coating $(n=0.645+5.029 \mathrm{i}$ at the operating wavelength $\lambda=532 \mathrm{~nm}$ ). The inner silica cone 
Fig. 1 Sketch of the fully metalcoated probe with spiral corrugation: a 3D representation, b projection on the $x y$ plane. The different mutual positions of the corrugation and the linearly polarised modes are created by clockwise (negative angles) and counter-clockwise (positive angles) rotations of the corrugation around the $z$ axis

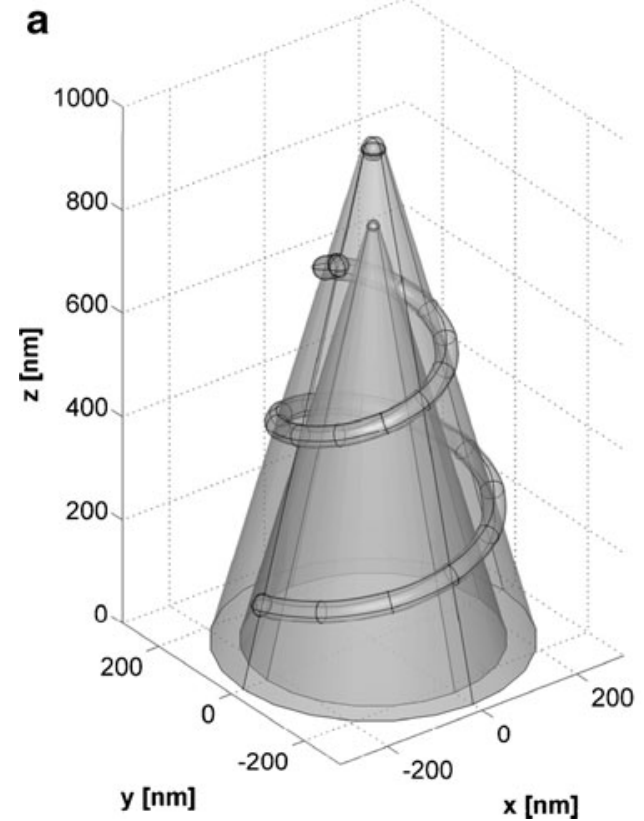

b

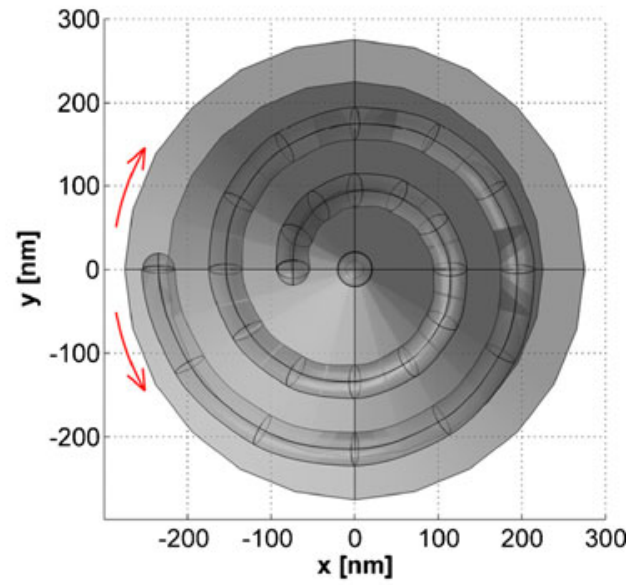

radius is $225 \mathrm{~nm}$, whereas the metallic hollow cone outer radius is $275 \mathrm{~nm}$. Both cones have an apex angle of $30^{\circ}$ and are rounded, with the radii of curvature amounting to 10 and $20 \mathrm{~nm}$, respectively. The modelling domain is a $1.6 \mu \mathrm{m}$ high cylinder with radius $1 \mu \mathrm{m}$. The spiral corrugation is formed by joining a tapered helix-shaped 3D object (with circular cross-section of radius $r$ ) with two hemispherical terminations (having the same radius $r$ ). In each point, the centre of the circular cross-section is located along the outer metal surface, so that the spiral winding appears as a semicircular spiral corrugation. The spiral is placed between 150 and $750 \mathrm{~nm}$ along the $z$ direction and the pitch along $z$ is $300 \mathrm{~nm}$. The size of the computational domain and the spiral parameters were chosen as a trade-off between the need to create an effective asymmetry and the computational cost. In fact, the nanoscale modelling of SNOM probes is computationally challenging. Moreover, in our case, the presence of an asymmetry rules out the reduction of the problem complexity through the use of two-dimensional (2D) approximations or the body of revolution finite difference time domain method as done for axisymmetric structures [9, 25]. A full 3D analysis becomes mandatory: the use of unstructured grids in the finite element method enables the treatment of such complicated geometries. The spiral corrugation can take on the form of either a groove, i.e. it is filled with air, or a bump, corresponding to metal filling. We have also investigated the effect of metal oxide filling. The computational process consists of two steps. First, a 2D analysis is run to calculate the eigenmodes supported at the input port in the plane at the bottom of the probe. Then, the first three eigenmodes are propagated through the probe in a 3D simulation. Second-order elements with minimum size of about $0.8 \mathrm{~nm}$ have been used. Simulations have been run on a 64-bit workstation with $32 \mathrm{~GB}$ of RAM.

In the following paragraphs, first we will examine the behaviour of the probe for different mutual orientations of the modes with respect to the spiral winding and variable $r$ and filling materials. Next, in order to highlight the advantages resulting from the introduction of an adirectional asymmetry like a spiral corrugation, we will estimate how the behaviour of probes based on directional asymmetries (namely cut tips and asymmetrically corrugated probes) depends on the polarisation direction.

\section{Variation in the Mutual Position of the Spiral Winding} and the Mode Orientation

As we were interested in assessing the behaviour of the tip under variable orientation of the linearly polarised mode with respect to the spiral corrugation, we carried out a set of simulations to account for all the possible relative positions. For this purpose, we considered $x$ and $y$ linearly polarised mode excitations (indicated from now on as $\mathrm{H}$ and $\mathrm{V}$ ) and examined the characteristics of the corresponding near-field distributions for different spiral orientations, by rotating the spiral winding. Due to the equivalence of the two orthogonally polarised modes, it was sufficient to rotate the corrugation over an angular range of $90^{\circ}$ around the $z$ axis to cover the whole spectrum of possible mutual positions. The zero rotation was arbitrarily set to be the one depicted in Fig. 1 (corresponding to the centre of the initial circular cross section at the extremes of the spiral winding located at $y=0$ ) and the structure was rotated from $-40^{\circ}$ to $45^{\circ}$ with a step of $5^{\circ}$. We have evaluated the characteristics of the near-field distribution close to the tip 
apex in terms of signal intensity and full width at half maximum (FWHM), which is related to resolution. The quantities we have chosen as figures of merit for the characterization of the sensitivity to polarisation direction are the maximum deviation of the FWHM and peak intensity from the average values as the orientation rotated. Smaller deviations suggest more uniform probe behaviour under variable polarisation direction.

Throughout the whole analysis, we have considered the behaviour of the axisymmetric probe (with the same geometric parameters except for the presence of the spiral corrugation) as a reference for comparison. For this reference structure, a localised hot spot with FWHM of $38 \mathrm{~nm}$ was obtained under radially polarised excitation for the square of the norm of the electric field in a transverse plane located at $10 \mathrm{~nm}$ from the tip apex, while broad twolobed distributions with average size of $400 \mathrm{~nm}$ and peak values about 50 times smaller than the one of the radial spot were observed in the same plane under linearly polarised excitation [22]. The size of the radial spot is mostly influenced by the diameter of the metal rounding at the apex [26]. In this paper, we have considered the intensity of the electric field in the same transverse plane for the tips with a spiral corrugation. In Fig. 2 the near-field intensity distributions for a spiral metal corrugation at zero rotation angle and radius $r=25 \mathrm{~nm}$ are illustrated. The values are normalised to the peak value of the reference probe under radially polarised excitation in order to better pinpoint the relative field strengths.

As expected, the presence of an adirectional asymmetry results in strong field localization for both the orthogonal linearly polarised modes. The ultrasmall spots are very similar in terms of both FWHM and peak value, even though their peak intensity is still five times lower than the one of the radial hot spot of the axisymmetric probe. However, as reported in the next paragraph, the intensity can be adjusted by varying either the filling material or the radius of the corrugation. Different physical mechanisms are responsible for field localization: the diverse forms of intercoupling between inner and outer SPPs and between SPPs and WGM induced by the spiral protrusion cause the electric fields associated with SPPs on the opposite sides of the probe not to have opposite phases any longer, a phenomenon leading to mutual cancellation at the tip apex for axisymmetric structures. The main advantage with respect to structures based on directional asymmetries lies in the fact that now both the linearly polarised modes oriented in orthogonal directions undergo similar processes and experience analogous shrinkage with comparable peak values, because the asymmetry involves all the spatial directions. It is worth to observe that the chirality of the spiral winding (that is whether it wraps the tip in clockwise or counter-clockwise direction) plays no role in this case: similar intensity distributions have been observed by changing this property.

In order to get deeper insight into the effects of the spiral winding, the FWHM and the peak value normalised to the peak achieved in the reference axisymmetric probe under radially polarised excitation have been calculated for the different angles encompassing all the possible mutual positions of the spiral and the mode orientation (Fig. 3).

As visible, the most striking feature is that only negligible fluctuations occur in the FWHM. Noticeably, the structure exhibits a spot size almost insensitive to variations in the mutual orientation, offering the potential for an easier implementation of high resolution microscopy. In fact, the FWHM is related to the eventual achievable resolution and hence its robustness with respect to misalignments in mode injection allows a marked simplification in experimental applications. Despite this tremendous advantage, the peak value is still slightly dependent on the mutual orientation: nonetheless, the maximum deviation from the average value calculated over all the mutual positions is less than $20 \%$, a value that could still be acceptable if the average value were sufficiently high for detection. Note that, due to the equivalence of the linearly polarised modes $\mathrm{H}$ and $\mathrm{V}$, just one average value is calculated for both of them as the average over all the rotation angles.

The degree of improvement becomes more apparent if compared with a similar analysis run on a structure with a directional asymmetry. In particular, we considered a structure with five asymmetric corrugations of radius $20 \mathrm{~nm}$, extended over an angular section of $160^{\circ}$. Corrugations could be either bumps (metal filling indicated by $m$ ) or grooves (air filling labelled as $a$ ) in the outer metal surface. We examined a probe with an alternation of metal/ air/air/metal/air (maama) starting from the bottom corrugation. While in [22] only a single mutual orientation of the corrugation with respect to the linearly polarised modes was considered (because the corrugations were located in such a way to create maximum asymmetry along $x$ and no asymmetry along $y$ ), in the present paper we rotated the corrugations in order to examine the sensitivity of the tip behaviour to the mutual orientation of the linearly polarised mode with respect to the asymmetry. We set the zero rotation angle to correspond to the position of maximum asymmetry along the $x$ direction and no asymmetry along $y$. Due to the symmetry of the corrugation with respect to $y=0$ at the zero rotation angle, it was sufficient to consider only the angular range between $0^{\circ}$ and $45^{\circ}$ to cover the whole range of possible mutual positions. Figure $4 \mathrm{a}, \mathrm{b}$ report the graphs obtained by rotating the asymmetrically corrugated tip maama.

For the sake of clarity, the field distributions for the $\mathrm{H}$ and $\mathrm{V}$ modes at the two extremes of the rotation are depicted in Fig. 4c,d. As evident, when the rotation is null, 
Fig. 2 Normalised near-field intensity distributions in a plane located at $10 \mathrm{~nm}$ from the apex of a fully metal-coated probe with a spiral metal corrugation of radius $r=25 \mathrm{~nm}$ (R stands for radially, $\mathrm{H}$ for $x$ - and $\mathrm{V}$ for $y$ linearly polarised excitation): a over a $600 \mathrm{~nm} \times 600 \mathrm{~nm}$ square area; $\mathbf{b}$ magnification over the $200 \mathrm{~nm} \times 200 \mathrm{~nm}$ square area enclosed in the white border in the upper row

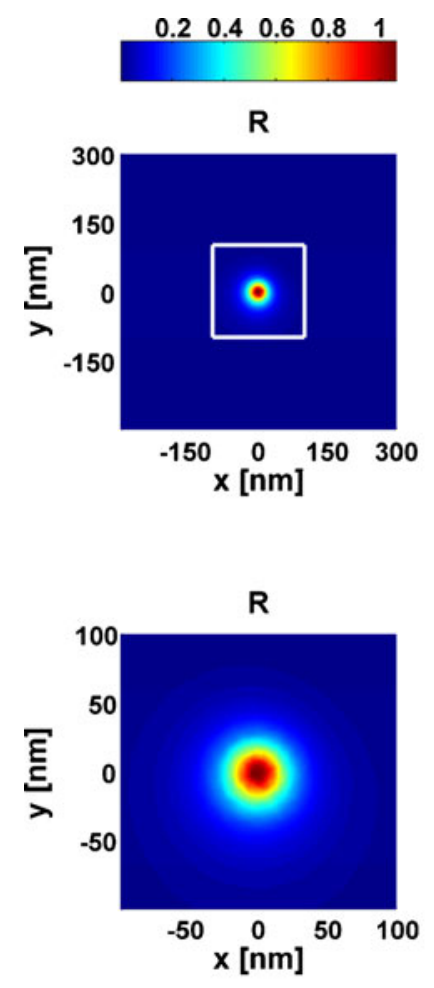

$\begin{array}{lll}0.05 & 0.1 & 0.15\end{array}$

$\begin{array}{llll}0.05 & 0.1 & 0.15 & 0.2\end{array}$

H

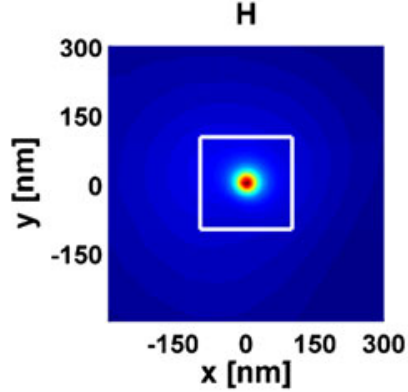

a

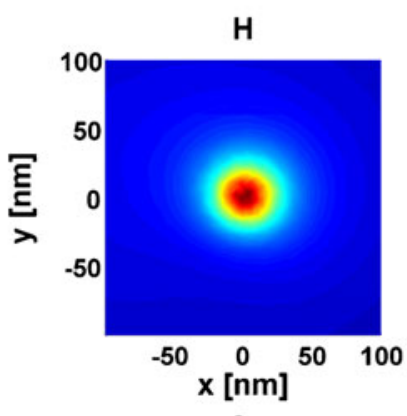

b

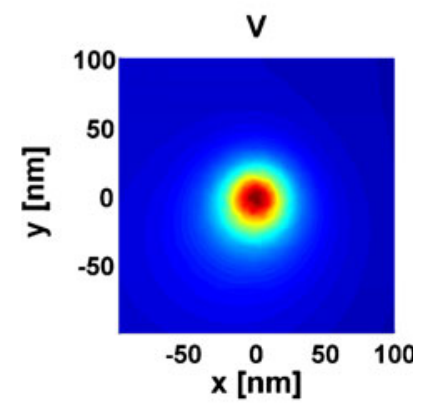

a strong asymmetry in just one direction $(x)$ is present, giving rise to strong field localization with high peak values under $\mathrm{H}$ excitation, while the $\mathrm{V}$ mode does not "feel" any asymmetry and exhibits a broad and weak distribution. As the corrugations are rotated, the asymmetry along $x$ is

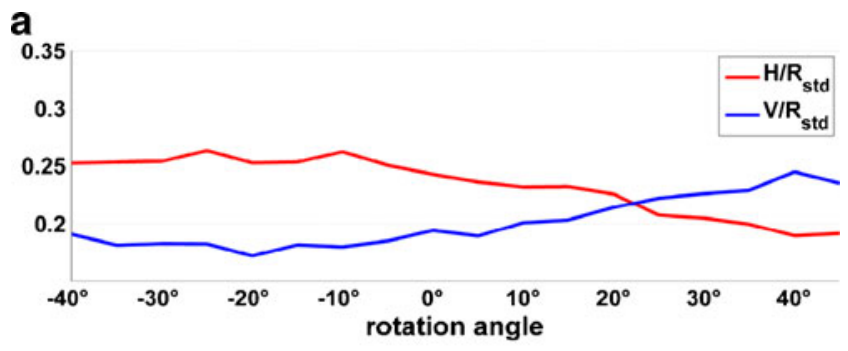

b

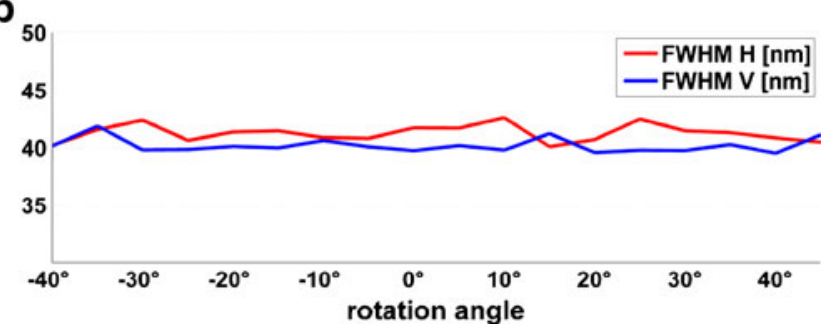

Fig. 3 Behaviour of the probe with spiral metal corrugation $(r=$ $25 \mathrm{~nm}$ ) for different mutual orientations of the corrugation and the linearly polarised mode: a comparison of the peak value with respect to the one of the standard reference probe under radially polarised excitation (denoted by $\mathrm{R}_{\text {std }}$ ); $\mathbf{b}$ FWHM reduced, while an asymmetry along $y$ appears and gradually increases. At the same time, the $\mathrm{H}$ distribution starts to decline in peak value, while the $\mathrm{V}$ distribution shrinks in size and climbs in peak value. At $45^{\circ}$, the asymmetry perceived by both the modes is the same and the corresponding distributions are roughly identical. In contrast to the spiral winding, the variation of the probe behaviour with relative alignment is radical, as shown also by the maximum deviation from the average value of the spot size and the peak value amounting to about $375 \%$ and $90 \%$, respectively.

Variation in Material and Geometric Parameters and Comparison with Directional Asymmetries

Both the FWHM and the peak value can be tuned and optimised by changing the characteristics of the spiral corrugation. As anticipated, an indentation can be used instead of a metal protrusion, that is the metal can be carved in a spiral shape, or, as an alternative, another dielectric such as aluminium oxide $(n=1.54)$ can fill the spiral corrugation. In fact, the coupling between surface modes at two adjacent metal/dielectric interfaces can be modulated by changing the indices of refraction of the dielectric materials [25]. Additionally, the radius of the spiral $r$ can be changed to get stronger or weaker asymmetries. This geometric parameter was varied from 15 to $30 \mathrm{~nm}$ with a step of $5 \mathrm{~nm}$. More specifically, for each structure based on a particular combination of filling material and radius $r$, we 


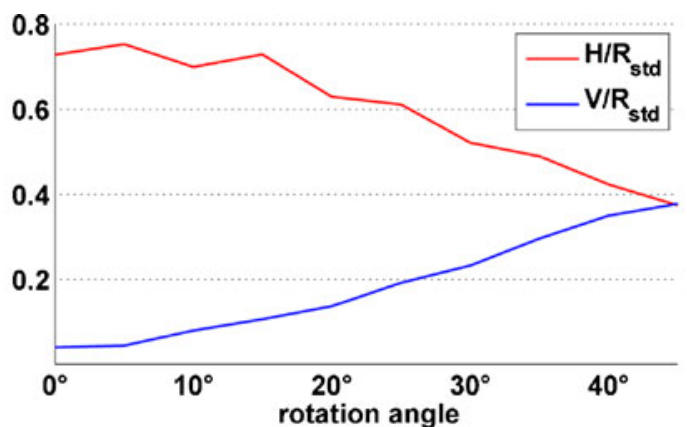

a
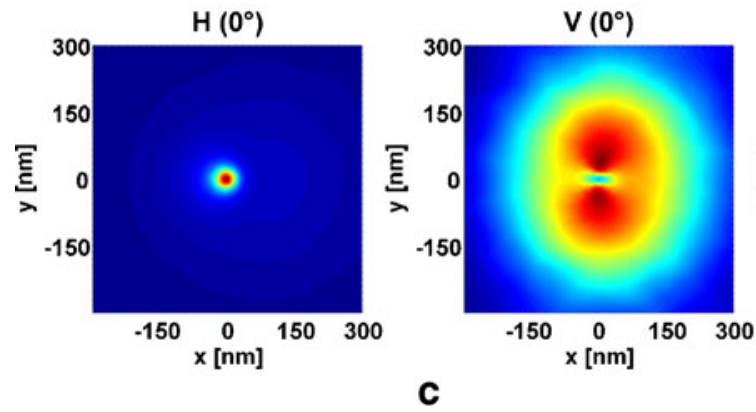

Fig. 4 Behaviour of the maama probe for different mutual orientations of the corrugation and the linearly polarised mode: a comparison of the peak value with respect to the one of the standard reference

undertook an analysis similar to the one shown in the previous paragraph for the spiral metal corrugation of radius $r=25 \mathrm{~nm}$, that is we rotated the corrugation from $-40^{\circ}$ to $45^{\circ}$ with a step of $5^{\circ}$. Then, we calculated the average value of the peak ratio and the FWHM for the linearly polarised distributions over all the rotated positions, as explained in the previous paragraph, and considered these values together with the maximum deviation from the corresponding average as figures of merit for comparison. The results for the three different filling materials as a function of the radius $r$ are reported in Fig. 5.

A global inspection of the graphs reveals that, when the asymmetry is small, the coupling mechanisms are less efficient and the field localization is less effective, giving rise to bigger spot sizes and lower peak intensities. As the asymmetry becomes stronger, the shrinkage in the field distributions gets more remarkable and the FWHM drops to values comparable to the size of the radial spot of the axisymmetric tip (which, as stated earlier, is mostly influenced by the diameter of the metal rounding at the apex). At the same time, the peak value increases as a result of improved coupling mechanisms. The better field localization is the outcome of a stronger asymmetry due to larger $r$ values. However, differently from the case of directional asymmetries, these results are achieved irrespective of the mutual orientation of the linearly polarised mode and the asymmetry introduced in the structure, as confirmed by the data about the maximum deviation. The deviation in

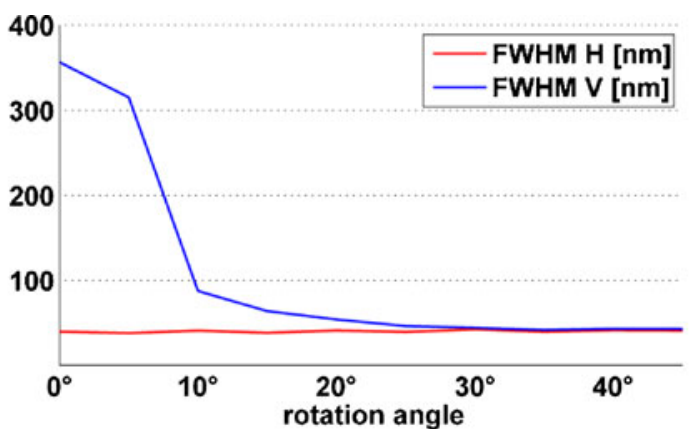

b
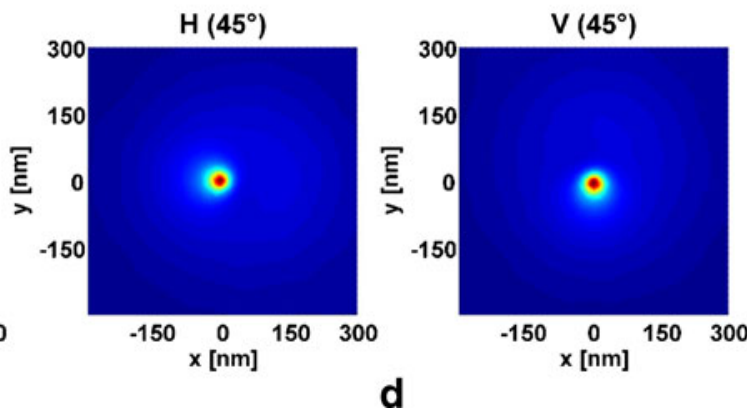

probe under radially polarised excitation (denoted by $\mathrm{R}_{\text {std }}$ ); b FWHM; c near-field distributions for the two linearly polarised modes at $0^{\circ}$; d near-field distributions for the two linearly polarised modes at $45^{\circ}$

FWHM dips as the radius increases until it reaches a point where the spot size can be considered almost constant with a variation in the reciprocal position. Although the peak value shows an opposite trend with a change of the spiral radius, the maximum deviations are still tolerable, especially if combined with a rise in the average value occurring when metal is replaced by air or, even better, by aluminium oxide. The behaviour of the probe as a function of the filling material can be explained as a result of better coupling mechanisms between inner and outer SPPs occurring when air is substituted with metal oxide, because the coupling of surface modes at two adjacent metaldielectric interfaces becomes more efficient when the indices of refraction of the two dielectrics are closer [27].

To better highlight the enhanced robustness of the spiral asymmetry against misalignments, we evaluated the behaviour of probes based on various forms of directional asymmetries with different geometric parameters as a function of the relative orientation between the $\mathrm{H}$ and $\mathrm{V}$ modes and the asymmetry itself. The structures we examined are an asymmetrically corrugated probe with four different configurations and a cut probe with three different sets of geometric parameters [22, 23]. Both tip categories are sketched in Fig. 6.

We labelled all the asymmetrically corrugated structures after the initial of the filling material starting from the bottom corrugation ( $a$ stands for air, $m$ for metal and $o$ for aluminium oxide). The angular section covered by the 
Fig. 5 Characteristics of the near-field intensity distributions for variable filling material and variable radius of the spiral corrugation under linearly polarised excitation ( $\mathrm{H}$ and $\mathrm{V})$ : a average value of the ratio between the peak value for the probe with spiral corrugation and the one of the standard reference probe under radially polarised excitation; $\mathbf{b}$ average value of the FWHM; c maximum deviation from the average value of the ratio between the peak value for the probe with spiral corrugation and the one of the standard reference probe under radially polarised excitation; $\mathbf{d}$ maximum deviation from the average value of the FWHM
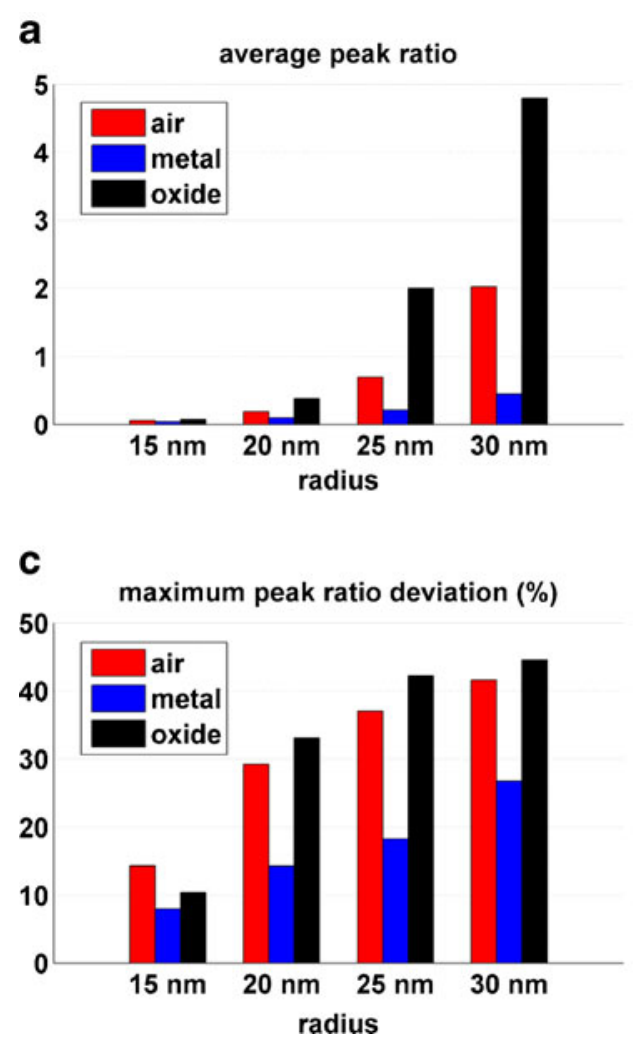
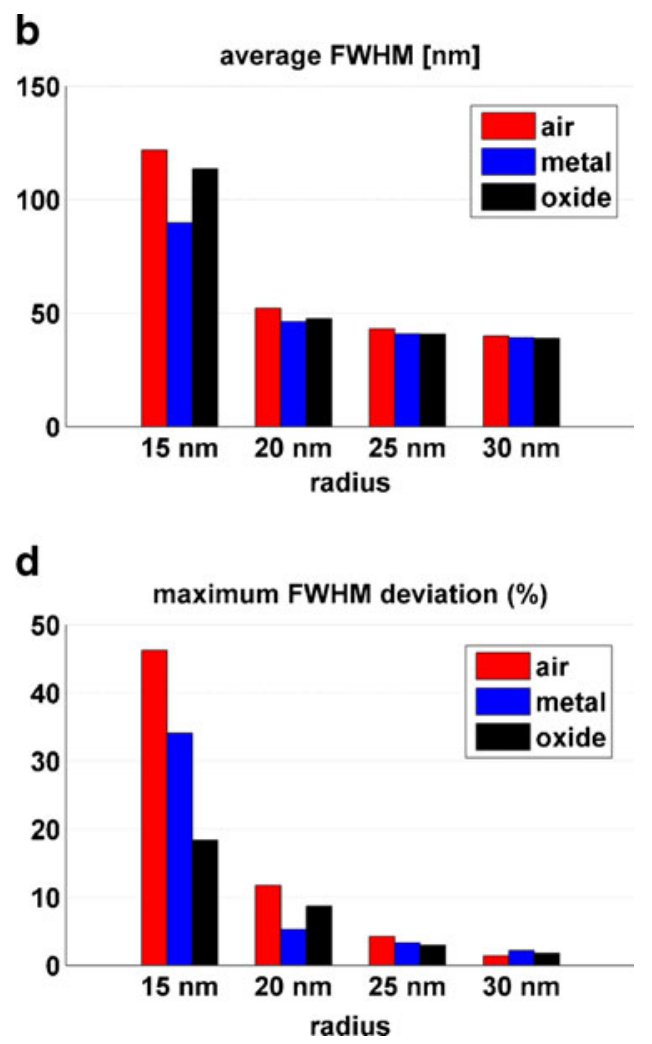

corrugation (opening angle) was $160^{\circ}$ in all the cases with the exception of the structure called amama $130^{\circ}$. As to the cut probe, the configurations called cut $30^{\circ}$ and cut $50^{\circ}$ refer to probes with the new apex after the cut located at $816 \mathrm{~nm}$ from the bottom of the computational domain and cut angles (measured between a plane orthogonal to the axis of the probe and the plane of the cut) of $30^{\circ}$ and $50^{\circ}$, respectively. The structure denoted as cut $766 \mathrm{~nm}$ has a cut height of $766 \mathrm{~nm}$ and a cut angle of $30^{\circ}$. These structures were studied in the original papers only for the case of maximum asymmetry along $x$ and no asymmetry along $y$ and, therefore, for a single mutual orientation of the
Fig. 6 Sketch of two forms of directional asymmetries: a cut probe, $\mathbf{b}$ tip with asymmetric corrugations

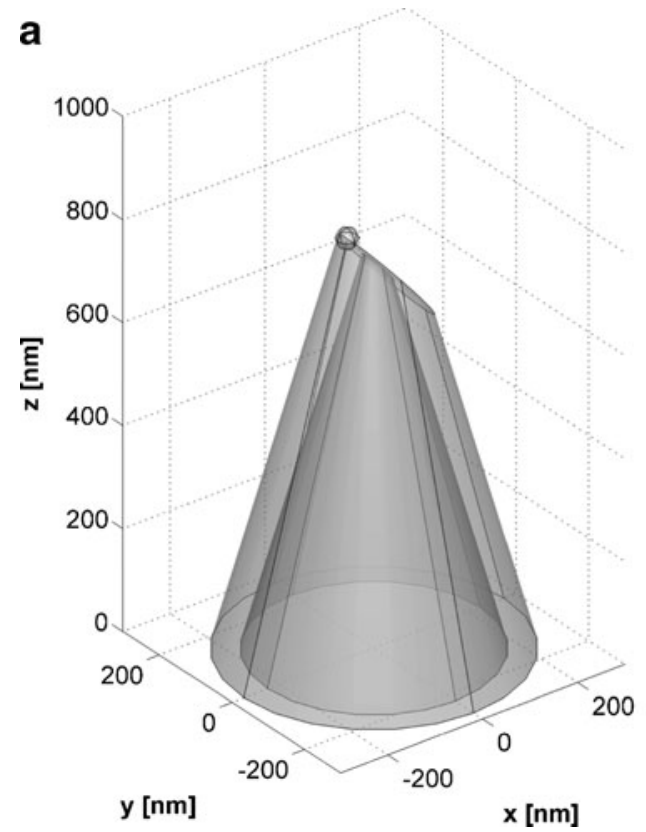

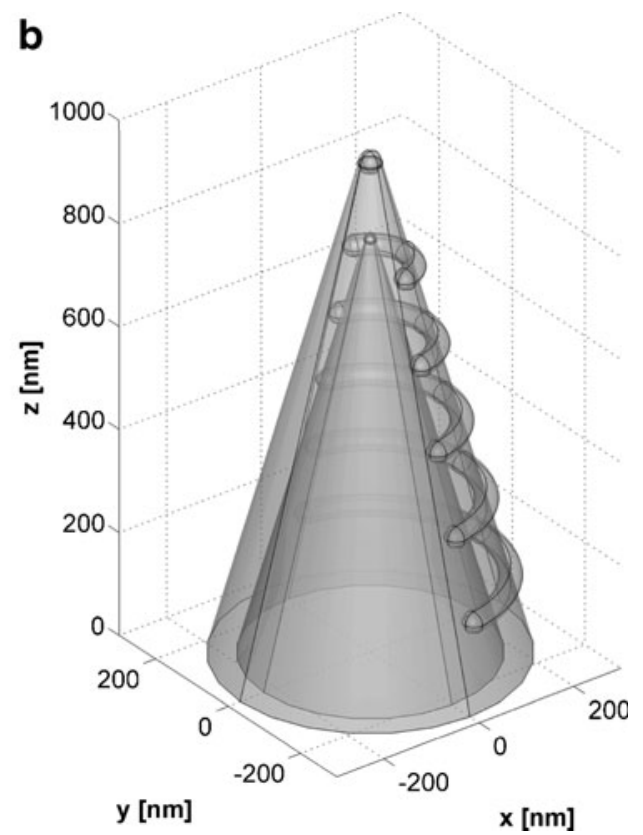


asymmetry with respect to the modes. In the current paper, the sensitivity to polarisation direction was studied by rotating each asymmetric structure from $0^{\circ}$ to $45^{\circ}$ with a step of $5^{\circ}$, with $0^{\circ}$ corresponding to maximum asymmetry along $x$ and no asymmetry along $y$ for both the cut tips and the asymmetrically corrugated probes. As we are mainly interested in the sensitivity to mode orientation, we report only the maximum deviation in the peak ratio and in the spot size from the average value over all the rotated positions (Fig. 7).

Variations of more than $90 \%$ in the peak value and, more importantly, stronger than $280 \%$ in the spot size are reported. The different sets of material and geometrical parameters considered show that such strong variations do not depend on how much the structures are optimised, but rather in their intrinsic directional nature. Aligning the input polarisation in a wrong way implies degradation not only of peak intensity, but also of resolution. On the contrary, the use of an adirectional asymmetry waives any bond on the input polarisation alignment.

\section{Conclusions}

An impressive simplification of the mode injection procedure in fully metal-coated probes can ensue from the introduction of an adirectional asymmetry, that is an asymmetry covering the whole angular range of the tip and devoid of an axis of symmetry. The actual structure here proposed as a proof of concept is a spiral corrugation. The lack of a preferential spatial direction allows the achievement of field localization for any mutual orientation of the spiral with respect to the linearly polarised mode.

The rate of improvement consequent upon the use of an adirectional asymmetry instead of directional ones has been estimated by comparing the orientation-dependent behaviour of structures based on the two different forms of asymmetries. The maximum deviation in spot size plummets from values between $280 \%$ and $500 \%$ for directional asymmetries to values of about $45 \%$ in the worst case for spiral adirectional asymmetry and even below $2 \%$ for proper choices of spiral parameters; likewise, the maximum variation in peak intensity falls from above $90 \%$ for directional asymmetries to below $45 \%$ for an adirectional asymmetry. Hence, the creation of a hot spot whose size is almost independent of the orientation of the linear polarisation and whose peak value changes only within a small range not only allows one to avoid the use of the radially polarised mode, but also permits not to care about the alignment of the linearly polarised mode with respect to the asymmetry. Requirements on alignment are correspondingly significantly relaxed.

The practical impact of such a result in experimental applications can be appreciated when considering the role played by the orientation of the linearly polarised mode in the images recorded by asymmetric tips based on a tip-onaperture and on a monopole antenna grown on the rim of an aperture tip [28-30]. For example, in [29, 30], dramatic variations in the imaging of fluorescent molecules were observed both in resolution and signal intensity by rotating the linearly polarised mode from the direction along which the metal protrusion is present to the orthogonal one, highlighting the strong influence of polarisation and the need to excite a suitably polarised mode. However, the control of the direction of the input polarisation is not an easy task especially in fibre-based tips due to the fact that the actual position of the asymmetry is unknown when the tip is mounted on the microscope and also because of the rotation of the incident light polarisation within the probe: in a tip-on-tip analysis of an asymmetric probe, the effective polarisation direction close to the asymmetry was determined a posteriori by observing the field distribution close to the tip apex and comparing it to simulation results [31]. The lack of accurate knowledge about the mutual orientation between the polarisation direction and the asymmetry would hinder a systematic application of such probes. The use of a fully metal-coated probe with adirectional asymmetries could potentially guarantee more uniform performance independently of the alignment.
Fig. 7 Characteristics of the near-field intensity distributions for directional asymmetries under linearly polarised excitation ( $\mathrm{H}$ and $\mathrm{V}$ ): a maximum deviation from the average value of the ratio between the peak value for the different directional asymmetries and the one of the standard reference probe under radially polarised excitation; $\mathbf{b}$ maximum deviation from the average value of the FWHM a

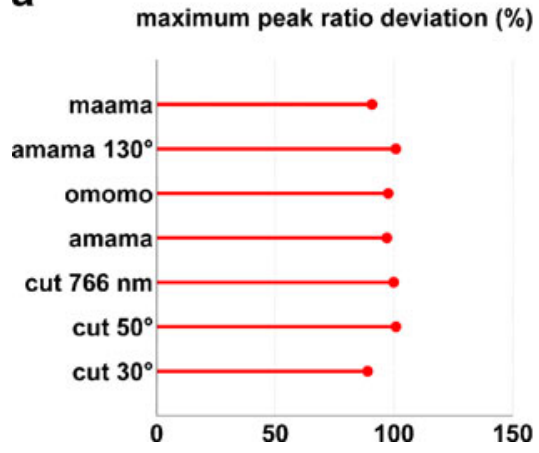

b

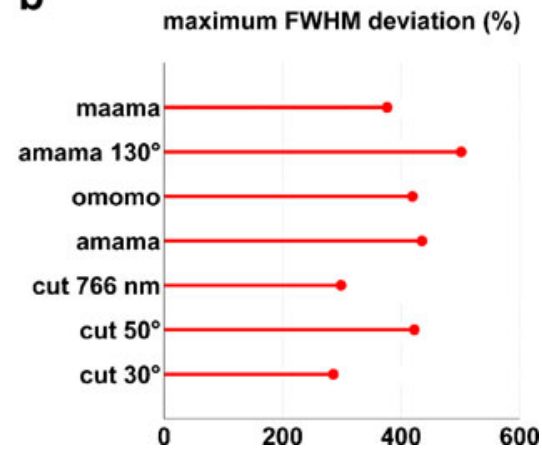


Although the spiral corrugation proposed in this work was conceived mostly as a proof of concept to explore the implications of the use of an adirectional asymmetry, an investigation of the effects of geometric and material parameters was carried out to show possible routes for optimization. Stronger asymmetries extended to larger tip sections originate better field localization. An extension of the computational domain could allow the study of the effects of other parameters, like the period, the number of turns and the shape of the corrugation; however, computational limits amply restrict the range of parameters that can be investigated.

Even though the fabrication of such a corrugation with a viable and reproducible method appears challenging, we have to point out that spiral lenses and gratings have been produced on planar structures [32, 33] and spiral fibre gratings have been fabricated for mode couplers [34]. Moreover, corrugations on tapered tips have been realised using focused ion beam and helically grooved metal wires, obtained by wrapping a wire around a cylindrical core, have been fabricated for terahertz SPP guiding applications [35, 36]. Even a more challenging structure based on a 3D array of free-standing metal helices has been realised for midinfrared frequencies [37]. In the wake of these encouraging results, we are confident that a spiral conical corrugation could be produced as well. Should this be possible, a characterization, based for example on tip-on-tip measurements $[19,31]$, could be carried out to validate our results.

Beyond the actual feasibility of this specific structure, the outcomes of our studies represent an interesting starting point and a clue to the design of more practical adirectional asymmetries, such as simpler modifications arranged in a spiral-like fashion. As a matter of fact, the use of adirectional asymmetries could bring about a staggering simplification and improvement in experimental applications.

Acknowledgements The authors gratefully acknowledge the support of the Swiss National Science Foundation (project number 200021115895).

Open Access This article is distributed under the terms of the Creative Commons Attribution Noncommercial License which permits any noncommercial use, distribution, and reproduction in any medium, provided the original author(s) and source are credited.

\section{References}

1. Novotny L, Hecht B (2006) Principles of nano-optics. Cambridge University Press, Cambridge

2. Antosiewicz TJ, Marciniak M, Szoplik T (2008) On SNOM resolution improvement. In: Sibilia C, Benson TM, Marciniak M, Szoplik T (eds) Photonic crystals: physics and technology. Springer, Milan, pp 217-238
3. Mononobe S (2005) Near-field optical fiber probes and the imaging applications. In: Ohtsu M (ed) Springer series in optical science-progress in nano-electro-optics III Springer Volume 96. Springer, Berlin, pp 1-56

4. Bouhelier A (2006) Field-enhanced scanning near-field optical microscopy. Microsc Res Tech 69:563-579

5. Girard C, Dereux A (1996) Near-field optics theories. Rep Prog Phys 59:657-699

6. Girard C (2005) Near field in nanostructures. Rep Prog Phys 68:1883-1933

7. Pohl D, Denk W, Lanz M (1984) Optical stethoscopy: image recording with resolution $\lambda / 20$. Appl Phys Lett 44(7):651653

8. Lewis A, Isaacson M, Harootunian A, Murray A (1984) Development of a $500 \AA$ spatial resolution light microscope: I. light is efficiently transmitted through $\lambda / 16$ diameter apertures. Ultramicroscopy 13(3):227-231

9. Liu L, He S (2005) Design of metal-cladded near-field fiber probes with a dispersive body-of-revolution finite-difference time-domain method. Appl Opt 44(17):3429-3437

10. Ding W, Andrews SR, Maier SA (2007) Internal excitation and superfocusing of surface plasmon polaritons on a silver-coated optical fiber tip. Phys Rev A 75:063822

11. Frey HG, Bolwien C, Brandenburg A, Ros R, Anselmetti D (2006) Optimized apertureless optical near-field probes with $15 \mathrm{~nm}$ optical resolution. Nanotechnology 17(13):3105-3110

12. Zeh C, Spittel R, Unger S, Opitz J, Köhler B, Kirchhof J, Bartelt H, Eng LM (2010) Polarization mode preservation in elliptical index tailored optical fibers for apertureless scanning near-field optical microscopy. Appl Phys Lett 97:103108

13. Chen W, Zhan Q (2007) Numerical study of an apertureless near field scanning optical microscope probe under radial polarization illumination. Opt Express 15(7):4106-4111

14. Janunts NA, Baghdasaryan KS, Nerkararyan KV, Hecht B (2005) Excitation and superfocusing of surface plasmon polaritons on a silver-coated optical fiber tip. Opt Commun 253(1-3):118-124

15. Bouhelier A, Renger J, Beversluis MR, Novotny L (2003) Plasmon-coupled tip-enhanced near-field optical microscopy. J Microsc 210(Pt 3):220-224

16. Descrovi E, Vaccaro L, Aeschimann L, Nakagawa W, Staufer U, Scharf T, Herzig HP (2005) On the coupling and transmission of transverse and longitudinal fields into fully metal-coated optical nano-probes. Proc SPIE 5736:96-104

17. Descrovi E, Vaccaro L, Aeschimann L, Nakagawa W, Staufer U, Herzig HP (2005) Optical properties of microfabricated fullymetal-coated near-field probes in collection mode. J Opt Soc Am A 22(7):1432-1441

18. Bouhelier A, Novotny L (2007) Near-field optical excitation and detection of surface plasmons. In: Brongersma ML, Kik PG (eds) Surface plasmon nanophotonics, Springer series in optical science-volume 131. Springer, Berlin, pp 139-154

19. Tortora P, Descrovi E, Aeschimann L, Vaccaro L, Herzig HP, Dändliker R (2007) Selective coupling of $\mathrm{HE}_{11}$ and $\mathrm{TM}_{01}$ modes into microfabricated fully metal-coated quartz probes. Ultramicroscopy $107(2-3): 158-165$

20. Nakagawa W, Vaccaro L, Herzig HP (2006) Analysis of mode coupling due to spherical defects in ideal fully metal-coated scanning near-field optical microscopy probes. J Opt Soc Am A 23(5):1096-1105

21. Nakagawa W, Vaccaro L, Herzig HP, Hafner C (2007) Polarization mode coupling due to metal-layer modifications in apertureless near-field scanning optical microscopy probes. J Comput Theor Nanosci 4(3):692-703

22. Lotito V, Sennhauser U, Hafner C (2010) Effects of asymmetric surface corrugations on fully metal-coated scanning near field optical microscopy tips. Opt Express 18(8):8722-8734 
23. Lotito V, Sennhauser U, Hafner C (2010) Finite element analysis of asymmetric scanning near field optical microscopy probes. J Comput Theor Nanosci 7(8):1596-1609

24. Quong MC, Elezzabi AY (2007) Offset-apertured near-field scanning optical microscope probes. Opt Express 15(16):10163-10174

25. Antosiewicz TJ, Wróbel P, Szoplik T (2010) Performance of scanning near-field optical microscope probes with single groove and various metal coatings. Plasmonics. doi:10.1007/s11468-010-9163-6

26. Vaccaro L, Aeschimann L, Staufer U, Herzig HP, Dändliker R (2003) Propagation of the electromagnetic field in fully coated near-field optical probes. Appl Phys Lett 83(3):584-586

27. Janunts NA, Nerkararyan KV (2001) Modulation of light radiation during input into a waveguide by resonance excitation of surface plasmons. Appl Phys Lett 79(3):299-301

28. Frey G, Keilmann F, Kriele A, Guckenberger R (2002) Enhancing the resolution of scanning near-field optical microscopy by a metal tip grown on an aperture probe. Appl Phys Lett 81 (26):5030-5032

29. Taminiau TH, Segerink FB, Moerland RJ, Kuipers LK, van Hulst NF (2007) Near-field driving of an optical monopole antenna. J Opt A Pure Appl Opt 9:S315-S321

30. Taminiau TH, Moerland RJ, Segerink FB, Kuipers L, van Hulst NF (2007) $\lambda / 4$ resonance of an optical monopole antenna probed by single molecule fluorescence. Nano Lett $7(1): 28-33$

31. Yatsui T, Kourogi M, Ohtsu M (1997) Highly efficient excitation of optical near-field on an apertured fiber probe with an asymmetric structure. Appl Phys Lett 71(13):1756-1758

32. Yang S, Chen W, Nelson RL, Zhan Q (2009) Miniature circular polarization analyzer with spiral plasmonic lens. Opt Lett 34 (20):3047-3049

33. Chen W, Abeysinghe DC, Nelson RL, Zhan Q (2010) Experimental confirmation of miniature spiral plasmonic lens as a circular polarization analyzer. Nano Lett 10:2075-2079

34. Lee KS (2001) Coupling analysis of spiral fiber gratings. Opt Commun 198:317-324

35. Ropers C, Neacsu CC, Raschke MB, Albrecht M, Lienau C, Elsaesser T (2008) Light confinement at ultrasharp metallic tips. Jpn J Appl Phys 47(7):6051-6054

36. Fernández-Domínguez I, Williams CR, García-Vidal FJ, MartínMoreno L, Andrews SR, Maier SA (2008) Terahertz surface plasmon polaritons on a helically grooved wire. Appl Phys Lett 93:141109

37. Gansel JK, Thiel M, Rill MS, Decker M, Bade K, Saile V, von Freymann G, Linden S, Wegener M (2009) Gold helix photonic metamaterial as broadband circular polarizer. Science 325:15131515 